\title{
Apparent Tolerance to the Acute Effect of Nicotine Results in Part from Distribution Kinetics
}

Herve C. Porchet, Neal L. Benowitz, Lewis B. Sheiner, and James R. Copeland

Division of Clinical Pharmacology and Experimental Therapeutics, Departments of Medicine and Laboratory Medicine, School of Medicine, School of Pharmacy, and Clinical Pharmacology Unit of the Medical Service, San Francisco General Hospital Medical Center, University of California, San Francisco, California 94143

\begin{abstract}
Persons exposed to nicotine develop tolerance to many of its effects. When heart rate and forearm venous blood concentration are plotted against time after intravenous administration of nicotine, a greater increase in heart rate is seen for a given nicotine concentration during the rising phase of nicotine concentrations than during the decreasing phase. This could be due to acute tolerance or to more rapid distribution of drug to effect site (brain) than to venous blood. To distinguish between these possibilities, six rabbits were given nicotine intravenously. Blood samples were taken from the internal jugular vein (reflecting brain concentration), and the femoral vein and artery. Brain concentrations peaked before femoral venous concentrations. Seven men received intravenous infusions of nicotine. Peripheral venous blood concentrations and cardiovascular responses were measured. Heart rate peaked before venous concentrations. A physiological kinetic model, fit to the rabbit data, was scaled to humans and used to predict "brain" concentrations in them. Heart rate and predicted brain concentrations peaked simultaneously. We conclude that the rapid development of tolerance to the cardioaccelerating effect of nicotine can be attributed, at least in part, to its distribution kinetics.
\end{abstract}

\section{Introduction}

Acute tolerance has been described for many drugs acting on the cardiovascular system (such as sympathomimetic amines $[1,2]$ or cardiac nitrates $[3,4])$ and on the central nervous system (such as alcohol [5-7]). Apparent acute tolerance to cardiovascular effects of nicotine has been reported in the course of a $30-\mathrm{min}$ infusion in people $(8,9)$. Acute tolerance is important in that it may limit the therapeutic effectiveness of a drug, or in the case of drugs of abuse, may be an important determinant of the pattern of drug self-administration. Evidence for acute tolerance includes the observation that, after an infusion when venous concentrations of a drug are falling, effects may be less at a given concentration than had previously been seen at the same concentration during the infusion, when concentrations were rising. However, differences in

Address correspondence to Dr. N. L. Benowitz, Building 30, 5th Floor, San Francisco, General Hospital Medical Center, 1001 Potrero Ave., San Francisco, CA 94110. 1987.

Received for publication 27 October and in revised form 2 June

J. Clin. Invest.

(C) The American Society for Clinical Investigation, Inc.

$0021-9738 / 87 / 11 / 1466 / 06 \$ 2.00$

Volume 80, November 1987, 1466-1471 blood concentration-effect relationships in rising and falling parts of the blood concentration-time curve can also be observed if concentrations of drug at the (venous) blood sampling site and site of effect equilibrate with arterial concentrations at different rates (10).

Most of the effects of nicotine at concentrations seen after exposure to tobacco smoke are mediated by actions on the brain and sympathetic nervous system (11). Increased heart rate, blood pressure, and elevated circulating catecholamine concentrations after nicotine reflect generalized sympathetic neural activation, mediated by the central nervous system (12). The site of action of nicotine at concentrations found in smokers is believed to be afferent chemoreceptors and/or central (brain) nervous system (13). Brain concentrations of nicotine, up to five times greater than corresponding venous blood concentrations (14), are achieved after intravenous drug administration due to rapid equilibrium of brain and arterial blood (15). The kinetics of nicotine at the arterial chemoreceptor are unknown, but this site also presumably equilibrates rapidly with arterial blood. We therefore hypothesized that the apparent development of acute tolerance to nicotine, inferred from the comparison of drug effects to venous concentrations, might be explained by the distributional disequilibrium between the central nervous system and venous blood, the former equilibrating more rapidly than the latter.

To examine this hypothesis, we conducted a study in rabbits and humans. Rabbits were used to quantify the distribution of nicotine to brain and peripheral tissues, as this requires multiple arterial and venous blood samples. Humans were used to assess the relationship between concentration and effect. In the human study, nicotine was infused to steady state, preceded by rapid or slow loading infusions. Cardiovascular responses and venous blood concentrations were measured. Using the distribution kinetics determined in the rabbit, appropriately scaled to the human, the pharmacodynamics of nicotine could be related to the time course of "brain", as well as forearm venous nicotine concentrations. We found that the time lag between venous blood concentrations and effect was not observed in the effect vs. brain concentration plot, implying that the rapid distribution of nicotine to brain may account for most of its apparent acute tolerance. An appreciation of distributional kinetics vs. true functional tolerance is relevant to our understanding of the pharmacodynamics of nicotine and of patterns of abuse of nicotine and other psychoactive drugs (16).

\section{Methods}

Subjects. Six male New Zealand white rabbits weighing between 3.0 and $4.2 \mathrm{~kg}$ (mean, $3.6 \mathrm{~kg}$ ) were used. Seven healthy men, regular cigarette smokers, $24-60 \mathrm{yr}$ of age (mean, 37), were investigated. The 
men smoked an average of 32.8 cigarettes/d (range, 30-40) with an average yield of $1.1 \mathrm{mg}$ of nicotine (range, $0.7-1.4$ ) and $16.7 \mathrm{mg}$ of tar (range, 8.3-23.1) per cigarette based on United States Federal Trade Commission smoking machine delivery data. Biochemical tests of liver function (serum glutamic-oxaloacetic transaminase, lactic dehydrogenase, alkaline phosphatase, total protein, and albumin) and kidney function (blood urea nitrogen and serum creatinine) were all within normal limits.

Experimental protocol. In the rabbits, after induction of anesthesia with ketamine $(150 \mathrm{mg} / \mathrm{kg})$ and diazepam $(1 \mathrm{mg} / \mathrm{kg})$ intramuscularly, four catheters were inserted. Catheters were placed in the right femoral vein for nicotine administration and in the left femoral vein, the right femoral artery, and the right internal jugular vein for blood sampling. An infusion of nicotine bitartrate, $25 \mu \mathrm{g}$ (nicotine base)/ $\mathrm{kg}$ per min, was given over $1 \mathrm{~min}$. Blood samples $(1 \mathrm{ml}$ each) for nicotine concentration were drawn simultaneously at the three sampling sites at $1,3,5$, $7,10,15,20,30,45$, and $60 \mathrm{~min}$. At the end of the experiment the animals were killed.

The human subjects were hospitalized for three consecutive days on the clinical research ward of the San Francisco General Hospital. They ate a normal diet except that caffeine-containing beverages and alcohol were prohibited. On the morning of the $2 \mathrm{nd}$ and $3 \mathrm{rd} d$, after an overnight fast and abstinence from smoking, an infusion of nicotine was administered. At least $1 \mathrm{~h}$ before nicotine was administered, intravenous catheters were inserted into an antecubital vein of one arm for injection of nicotine, and into a forearm vein of the other arm for blood sampling. Subjects remained in the recumbent position, except to void, during the experiment. Heart rate, blood pressure, and plethysmographic pulse wave amplitude were recorded by electrocardiogram (model 7 polygraph; Grass Instrument Co., Quincy, MA), Dinamap (model 845XT; Critikon, Tampa, FL), and photoplethysmograph (Vasculab model PPG13, MedaSonics, Inc., Mountain View, CA) for $30 \mathrm{~min}$ before, (minimum of five recordings) to obtain baseline values, and at frequent intervals during nicotine infusion. The photoplethysmograph was applied to the middle finger of one hand.

Nicotine bitartrate in sterile $0.9 \%$ sodium chloride was infused at constant rate by infusion pump. On one day the nicotine infusion rates were $17.5 \mu \mathrm{g}$ (nicotine base) $/ \mathrm{kg}$ per min from 0 to $1.5 \mathrm{~min}, 1.75 \mu \mathrm{g} / \mathrm{kg}$ per min from 1.5 to $30 \mathrm{~min}$, and then $0.35 \mu \mathrm{g} / \mathrm{kg}$ per min from 30 to $180 \mathrm{~min}$. On the other day the nicotine infusion rates were $2.5 \mu \mathrm{g} / \mathrm{kg}$ per min from 0 to $30 \mathrm{~min}$, and then $0.35 \mu \mathrm{g} / \mathrm{kg}$ per min from 30 to 180 $\mathrm{min}$. These rates were chosen to achieve the same steady state blood concentrations of nicotine after $\sim 1 \mathrm{~h}$. The rates were calculated by using the method of Wagner (17). Blood samples $(3 \mathrm{ml} \mathrm{each})$ for nicotine concentration were drawn at frequent intervals during the time of the nicotine infusion and for 180 min afterwards.

Nicotine analysis. Blood samples were assayed for nicotine concentrations by gas chromatography using nitrogen-phosphorus detection (18), modified for use with capillary columns.

Pharmacodynamic measurements. Increases in heart rate and blood pressure were defined as the difference between baseline (the mean of five predrug measurements) and the value measured during nicotine infusion. The plethysmographic effect was defined as the difference of amplitude between baseline and the value measured during nicotine infusion, expressed as a percentage of the baseline value (19). A $100 \%$ effect corresponds to complete vasoconstriction with a flat photoplethysmographic recording, defined as the maximal effect $\left(E_{\max }\right){ }^{1}$ This method assesses changes in blood flow to skin (20).

Pharmacokinetic analysis. The blood concentrations of nicotine measured in the rabbits were fitted to a three compartment mammillary model (Fig. 1) using extended least squares regression (21). Compartment one is identified with arterial blood; compartment two, with musculoskeletal and visceral tissues; and compartment three, with

1. Abbreviations used in this paper: $\mathrm{E}_{\max }$, maximal effect; $\mathrm{PC}$, partition coefficient; $\mathrm{PC}_{\text {brain }}$, brain-blood PC; $\mathrm{PC}_{\text {periph }}$, peripheral tissue-blood PC.

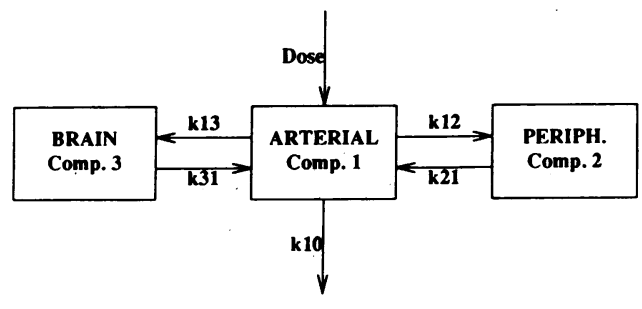

Figure 1. Three-compartment mammillary model used to fit blood concentrations of nicotine in rabbits. Compartments one, two, and three correspond to femoral artery, femoral vein, and internal jugular vein blood, respectively.

brain. Assuming equilibration of concentrations between blood leaving an organ and organ tissues, concentrations in compartments two and three are proportional to those in femoral and internal jugular blood, respectively. The following physiologic parametrization of the model was used: The apparent volume of distribution of each compartment is defined as equal to its real physical volume multiplied by the partition coefficient (PC) between this compartment and blood. The apparent volume of distribution of compartment one (arterial blood) is equal to total blood volume (its PC is 1). The apparent volume of distribution of compartment two is equal to total body volume minus brain volume multiplied by the peripheral tissue-blood PC ( $\left(\mathrm{PC}_{\text {periph }}\right)$. The apparent volume of distribution of compartment three is equal to brain volume multiplied by the brain-blood PC ( $\left.\mathrm{PC}_{\text {brain }}\right)$. The rate constants $k_{i j}$, connecting compartment $i$ to compartment $j$, are equal to the blood flow to compartment $j$ from compartment $i$ divided by the volume of distribution of compartment $i$. The elimination rate constant from compartment one is equal to total clearance divided by the volume of distribution of the central compartment. The physical volumes and blood flows to/from each compartment were obtained from the literature $(22,23)$ and are shown in Table I. Three parameters were estimated by the fitting procedure: total clearance and the two unknown $\mathrm{PC}$ ( $\mathrm{PC}_{\text {periph }}$ and $\left.\mathrm{PC}_{\text {brain }}\right)$. The parameter-estimation procedure fits the general triexponential equation for a classical three-compartment mammillary model with elimination from the central compartment to the mean of the experimental data of the six rabbits (24).

The same three compartment model used for the rabbit data, with the same parametrization, was used to fit the human venous blood concentrations of nicotine. Literature values were used for tissue volumes and blood flows (Table I) (25); total clearance was fixed to the value of dose divided by the observed area under the venous concentration curve from zero to infinity. The area under the venous concentration curve was calculated using the trapezoidal rule with extrapolation of the terminal portion to infinity. Extrapolation was done by dividing the nicotine concentration measured at $360 \mathrm{~min}$ by the slope of the linear regression of the log blood nicotine concentration vs. time from 210 to $360 \mathrm{~min}$ (data shown on Fig. 5). $\mathrm{PC}_{\text {brain }}$ were assumed to be identical in man and the rabbit. The only fitted parameter for the human model (in addition to clearance) is the $\mathrm{PC}_{\text {periph }}$.

Table I. Literature Values for Parameters of the Kinetic Model

\begin{tabular}{lrr}
\hline Parameter & Rabbit & Human \\
\hline Total body volume $(\mathrm{ml})$ & 3,600 & 70,000 \\
Total blood volume $(\mathrm{ml})$ & 225 & 5,600 \\
Brain volume $(\mathrm{ml})$ & 13 & 1,400 \\
Peripheral tissue volume $(\mathrm{ml})$ & 3,587 & 68,600 \\
Cardiac output $(\mathrm{ml} / \mathrm{min})$ & 770 & 5,400 \\
Brain blood flow $(\mathrm{ml} / \mathrm{min})$ & 10 & 750 \\
Peripheral blood flow $(\mathrm{ml} / \mathrm{min})$ & 760 & 4,650 \\
\hline
\end{tabular}




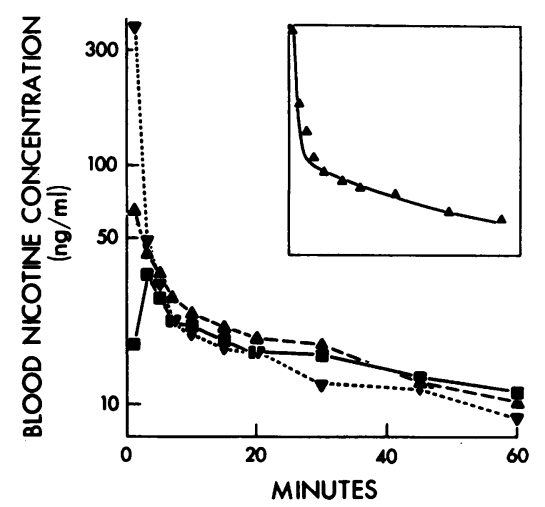
jugular venous data (triangles).

\section{Results}

Mean nicotine concentration vs. time curves for the six rabbits are shown in Fig. 2. The arterial and internal jugular nicotine concentrations were much higher than those in the femoral venous blood during the lst five min. The femoral venous concentrations increased continuously during the $1 \mathrm{st} 3 \mathrm{~min}$, whereas the two other concentrations decreased. The internal jugular concentration vs. time curve crossed the arterial concentration vs. time curve at four min, whereas the curve of the femoral venous concentration crossed that of the arterial concentration later at $8 \mathrm{~min}$. This indicates that equilibration between the brain and arterial blood is much faster than that between peripheral tissues and arterial blood. The values obtained for the model parameters after fitting these data are shown in Table II. That $k_{31}$ is 3.3 times greater than $k_{21}$ indicates that brain equilibrates with arterial blood 3.3 times faster than do the peripheral tissues. Whereas the three-compartment model used is only a crude approximation to reality, the fit to the jugular venous blood curve, the most crucial for our purposes (see below), is satisfactory (see Fig. 2 inset).

Nicotine infusions were well tolerated by all of the human subjects. Fig. $3 A$ shows the mean blood concentration vs. time curve obtained for each infusion. The curves are similar and almost superimposable except for the $1 \mathrm{st} 5 \mathrm{~min}$, where the increase in blood concentrations is steeper with the rapid

Table II. Values of the Parameters of the Kinetic Model

\begin{tabular}{lcc}
\hline Parameter & Rabbit & Human \\
\hline Total clearance $(\mathrm{ml} / \mathrm{min})$ & $55^{*}$ & $1,300^{*}$ \\
$\mathrm{PC}_{\text {brain }}$ & $0.779^{*}$ & 0.779 \\
$\mathrm{PC}_{\text {periph }}$ & $0.729^{*}$ & $1.44^{*}$ \\
$k_{12}\left(\mathrm{~min}^{-1}\right)$ & $3.370^{\ddagger}$ & $0.830^{\ddagger}$ \\
$k_{21}\left(\mathrm{~min}^{-1}\right)$ & $0.298^{\ddagger}$ & $0.047^{\ddagger}$ \\
$k_{13}\left(\mathrm{~min}^{-1}\right)$ & $0.044^{\ddagger}$ & 0.134 \\
$k_{31}\left(\mathrm{~min}^{-1}\right)$ & $0.987^{\ddagger}$ & 0.688 \\
$k_{31} / k_{21}$ & 3.312 & 14.638 \\
\hline
\end{tabular}

* Primary parameters obtained by fitting the data.

* Secondary parameters as computed from Table I values and the primary parameters.

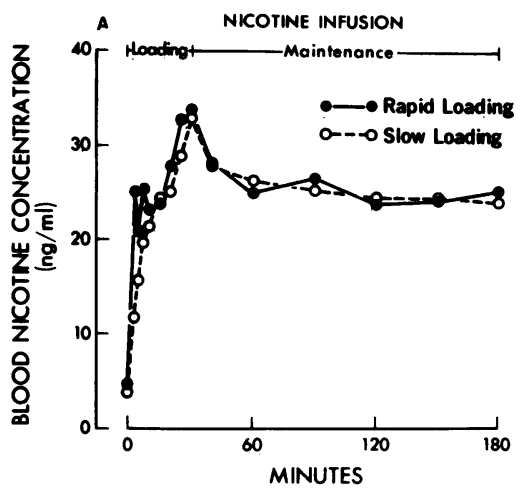

Figure 3. Nicotine results in seven subjects during two (rapid and slow) nicotine infusion protocols. Rapid infusion protocol was 17.5 $\mu \mathrm{g} / \mathrm{kg}$ per min from 0 to $1.5 \mathrm{~min}$ and $1.75 \mu \mathrm{g} / \mathrm{kg}$ per min from 1.5 to 30 $\mathrm{min}$. Slow infusion protocol was $2.5 \mu \mathrm{g} / \mathrm{kg}$ per $\mathrm{min}$ from 0 to $30 \mathrm{~min}$. Maintenance infusion rate for both protocols was $0.35 \mu \mathrm{g} / \mathrm{kg}$ per min from 30 to $180 \mathrm{~min}$. $(A)$

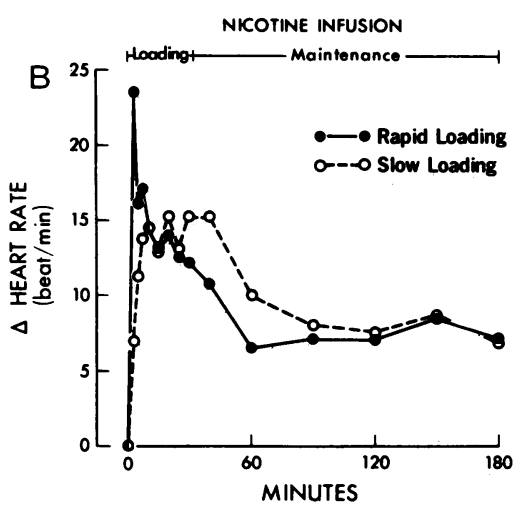

Mean blood nicotine concentration vs. time. The average coefficient of variation at the various points is $30 \%$ (range, 21-49\%). (B) Mean increase in heart rate vs. time. Infusion rates of nicotine are as in the legend to Fig. 3. The average coefficient of variation at the observation points is $60 \%$ (range, 38-100\%).

loading method of nicotine administration. The maximal forearm venous blood concentration was achieved at the same time $(30 \mathrm{~min}$ ) with comparable values, 33.6 and $33.0 \mathrm{ng} / \mathrm{ml}$ after the rapid and the slow loading infusions, respectively. The clearance of nicotine ws 1,323 and $1,260 \mathrm{ml} / \mathrm{min}$ for the fast and slow loading regimens (these values were determined by using the nicotine blood concentration measured up to $360 \mathrm{~min}$ ).

The mean changes in heart rate for the human subjects are depicted in Fig. 3 B. After the rapid loading infusion, heart rate increased quickly, reaching its maximum at $3 \mathrm{~min}$, followed by a decline to a plateau at $60 \mathrm{~min}$. In contrast, during the slower loading infusion, the heart rate increased more slowly, peaking at $30 \mathrm{~min}$. The effect then declined and the same heart rate plateau as for the rapid infusion was achieved after 90 min.

Fig. 4 shows the relationship between concentrations of nicotine in forearm venous blood and heart rate acceleration

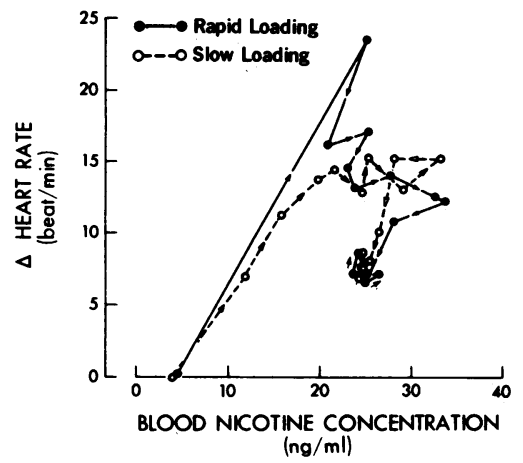

Figure 4. Average increase in heart rate plotted against the simultaneous mean forearm venous blood concentrations of nicotine for the two infusion protocols (see legend of Fig. 3). The points are connected in time order. 


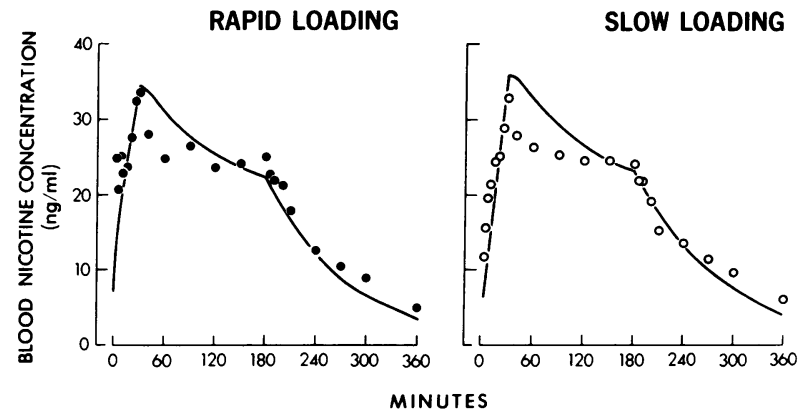

Figure 5. Fit of pharmacokinetic model (solid lines) to venous concentration data of humans (data as in Fig. $3 \mathrm{~A}$ ).

over time for the two different infusions. That the curves are not identical in the rising and falling parts of the curve in this sort of plot is termed hysteresis. A much greater effect was observed for a concentration of $\sim 25 \mathrm{ng} / \mathrm{ml}$ with the faster infusion than with the slower infusion. This concentration was achieved earlier with the rapid ( $3 \mathrm{~min})$ rather than the slow (15 min) loading infusion (Fig. $3 \mathrm{~A}$ ).

Using typical human tissue volumes and flows (Table I), the $\mathrm{PC}_{\text {brain }}$ from the rabbit and the $\mathrm{PC}_{\text {periph }}$ estimated from the mean human data, we estimate that nicotine equilibrates with the human brain $\sim 14$ times faster than with peripheral tissues $\left(k_{31}\right.$ is 14.6 times greater than $\left.k_{21}\right)$. The fit to the venous blood data shows some systematic error (see Fig. 5), but is used here only to establish the approximate $\mathrm{PC}_{\text {periph }}$ : detailed tracking of actual kinetics is not needed. Fig. 6 shows the predicted brain concentrations in humans using the fit and the approximate value of $k_{31}$ as scaled from the rabbit. Except for the $1 \mathrm{st} 5 \mathrm{~min}$, the brain nicotine concentration vs. time curves are quite similar for the rapid and slow loading infusions. After the fast infusion, the maximum concentration was achieved at $3 \mathrm{~min}$ and then fell rapidly. These curves are reasonably similar to the shape of the heart rate increase vs. time curves (Fig. $3 B$ ). When the increase in heart rate is plotted against the predicted brain concentration (Fig. 7), hysteresis is no longer observed. An apparently linear relationship between the predicted brain nicotine concentrations and the increase in heart rate is seen.

The three other pharmacodynamic effects (systolic and diastolic blood pressure and plethysmographic effect) show greater inter- and intraindividual variability. Except for the diastolic blood pressure, however, the maximum pharmacodynamic effect always occurs at the time of the maximum pre-

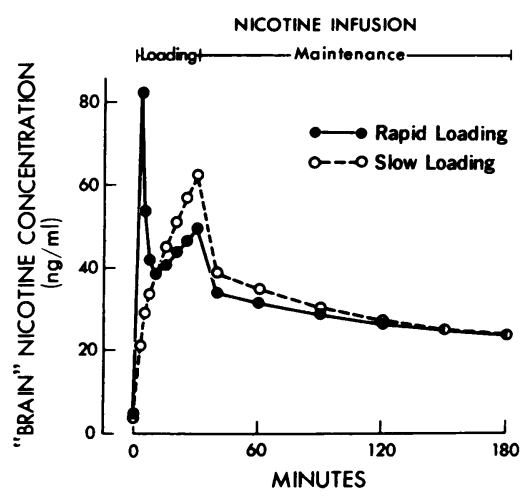

Figure 6. Predicted mean brain nicotine concentrations in seven subjects vs. time. Infusion rates of nicotine are described in the legend to Fig. 3.

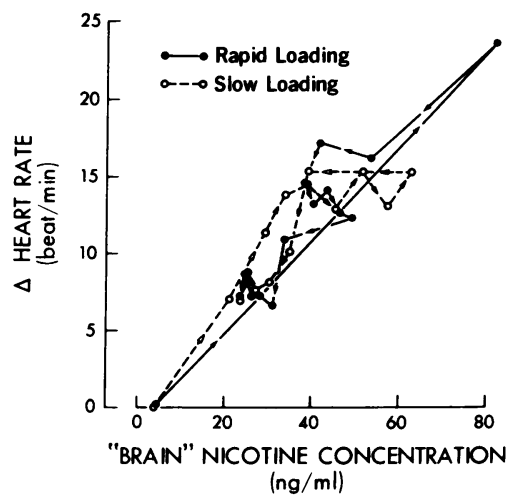

Figure 7. Average increase in heart rate vs. the simultaneous mean predicted brain concentration of nicotine for the two loading infusions. The points are connected in time order.

dicted brain concentration, not venous concentration, as shown in Table III.

\section{Discussion}

The main finding of this study is that the apparent development of acute tolerance to heart rate increase during nicotine infusion may be due partly, if not completely, to the distribution kinetics of the drug rather than to the rapid development of functional tolerance. In an acute experiment, such as the present one, development of tolerance is suggested by the observation that when effect is plotted against corresponding venous blood concentrations of nicotine, and the points are connected in time order, clockwise hysteresis is seen. That is, a smaller effect is associated with a given concentration of nicotine on the descending limb of the concentration vs. time curve than on the ascending limb. However such hysteresis might also be caused by the effect site, the brain, achieving equilibrium with arterial blood faster than the sampling site, the forearm venous blood.

Assuming a venous equilibration model, in which drug in the blood leaving an organ is in equilibrium with drug in the organ, the results of the rabbit experiment demonstrate a discrepancy between equilibration rates for brain, the presumed effect compartment, and venous blood. These findings are consistent with the results of the autoradiographic studies of Schmiterlow et al. (26) who showed that high concentrations of nicotine are present in the brain 5 min after intravenous injection in mice and that most of the nicotine has been cleared from the brain by $30 \mathrm{~min}$. Stalhandske (14) showed

Table III. Time to Reach Maximum Concentrations and Effects with the Two Infusion Regimens in Humans

\begin{tabular}{lll}
\hline & \multicolumn{2}{l}{ Peak time $($ min $)$} \\
\cline { 2 - 3 } Variable & Rapid loading & Slow loading \\
\hline Blood concentration & 30 & 30 \\
"Brain" concentration & 3 & 30 \\
Increase in heart rate & 3 & 30 \\
Percent E & 3 & 30 \\
Increase in systothysmographic & 5 & 30 \\
Increase in diastolic BP & 5 & 5 \\
\end{tabular}

${ }^{*} \mathrm{E}_{\max }$ is equal to $100 \%$ vasoconstriction. 
that intravenously injected ${ }^{14} \mathrm{C}$-nicotine is immediately taken up in the brain of mice, reaching a maximum concentration within $1 \mathrm{~min}$ after injection.

Our distributional model demonstrates that brain concentrations track drug effects over time nicely. The hysteresis loops collapse when the blood venous concentrations are replaced by predicted brain concentrations. Thus, apparent tolerance (clockwise hysteresis) can be explained by distribution kinetics alone, without invoking the development of functional tolerance, contrary to previous claims (27). The correspondence of time of all (save one) peak effects to the time of peak brain, but not venous, blood concentrations lends further support to this claim. A possible explanation for the early (anomalous) peak of the diastolic blood pressure with the slow loading infusion is that this is the maximal effect.

Our estimates of brain concentrations of nicotine involve several assumptions. We assume that the $\mathrm{PC}_{\mathrm{brain}}$ is the same in humans and in rabbits, that the blood flow to the various sites is constant (although nicotine induces many changes in cardiovascular homeostasis), and that the distribution of nicotine to tissues in the head other than the brain can be neglected. Even if these assumptions are imperfect, it is likely that, as in rabbits (Fig. 2) brain concentrations of nicotine in humans, too, will peak earlier after a rapid infusion, than do forearm venous concentration. Thus, at least some of the apparent acute tolerance to effects of nicotine that we observe must be a consequence of these distribution kinetics.

Rapid equilibration between arterial blood and the brain is a characteristic shared by other drugs of abuse, such as heroin and cocaine. Our model illustrates and quantitates the concept that the level in the brain and the magnitude of the psychoactive effects of such drugs will be greater when the drug is dosed rapidly. This explains why cocaine abusers prefer smoked (inhaled) cocaine to intranasal cocaine: the former is absorbed more rapidly. Our data also indicate that the magnitude and duration of effects of a dose of many drugs of abuse is determined in part by the distribution kinetics of the drug to the brain. This concept may explain the relatively low efficacy of nicotine gum when used in smoking cessation programs (28). In contrast to cigarette smoking, high brain concentrations cannot be achieved via the oral route because the rate of release of nicotine provided by the gum is insufficiently rapid, as the oral rate of absorption, to achieve high arterial and hence brain concentrations.

Our study has implications for the appropriate methodology for studying acute tolerance. We emphasize first that, when the concentration of a drug at the effect site itself cannot be measured, acute tolerance cannot be assessed by bolus-dose experiments alone (as by Chow et al., for example, in their study of cocaine effects [29]). A similar point has previously been made regarding ethanol $(30,31)$. To distinguish acute functional tolerance from distributional effects, steady state type experiments may be required. A decline in effect of a drug in the presence of a steady state blood concentration indicates functional tolerance as all body compartments are at equilibrium. However steady state may be difficult to achieve rapidly, and tolerance may have already developed by the time steady state is reached. In this case a decline in effect at steady state will not be observed. An alternative approach is to administer repeated bolus doses at relatively short time intervals. If tolerance develops, each successive dose should produce a smaller effect. Rosenberg et al. (9) and Russell et al. (32) have shown, in two different studies, that when two short bolus infusions of nicotine separated by $30 \mathrm{~min}$ or more are given to the same subject, the intensity of the response induced by the second infusion is smaller than that induced by the first. This finding is hard to explain by any mechanism other than functional tolerance and suggests that part of the clockwise hysteresis we see may be related to such tolerance. Our study does not address the development of the long-term tolerance to nicotine that has been demonstrated in animals and humans $(33,34)$.

\section{Acknowledgments}

Supported in part by United States Public Health grants GM 26691, DA 02277, DA 01696, and HL 29467. The studies were carried out in the General Clinical Research Center at San Francisco General Hospital Medical Center (RR-00083) with support by the Division of Research Resources, National Institutes of Health. Dr. Porchet was supported as a Fellow of the Swiss National Science Fund during the course of this work.

\section{References}

1. Harden, T. K. 1983. Agonist-induced desensitization of the beta-adrenergic receptor-linked adenylate cyclase. Pharmacol. Rev. 35:5-32.

2. Sibley, D. R., and R. J. Lefkowitz. 1985. Molecular mechanisms of receptor desensitization using the beta-adrenergic receptor-coupled adenylate cyclase system as a model. Nature (Lond.). 317:124-129.

3. Armstrong, P. W., and J. A. Moffat. 1983. Tolerance to organic nitrates: clinical and experimental perspectives. Am. J. Med. 74:73-84.

4. Leier, C. V. 1985. Nitrate tolerance. Am. Heart J. 110:224-232.

5. Aschan, G., M. Bergstedt, L. Goldberg, and L. Laurell. 1956. Positional nystagmus in man during and after alcohol intoxication. $Q$. J. Stud. Alcohol. 17:381-405.

6. Mellanby, E. 1919. Alcohol: its absorption into and disappearance from blood under different conditions. Medical Research Committee, Special Report Series No. 31, His Majesty's Stationary office, London.

7. Caspers, H., and G. Abele. 1956. Hirnelektrische untersuchungen zur frage der quantitativen beziehungen zwischen blutalkoholgehalt und alkohol-effekt. Deut. Z. gesamte Exp. Gerichtl. Med. 45:492509.

8. Benowitz, N. L., P. Jacob, III, R. T. Jones, and J. Rosenberg. 1982. Interindividual variability in the metabolism and cardiovascular effects of nicotine in man. J. Pharmacol. Exp. Ther. 221:368-372.

9. Rosenberg, J., N. L. Benowitz, P. Jacob, III, and M. Wilson. 1980. Disposition kinetics and effects of intravenous nicotine. Clin. Pharmacol. Ther. 28:517-522.

10. Galeazzi, R. L., L. Z. Benet, and L. B. Sheiner. 1976. Relationship between the pharmacokinetics and pharmacodynamics of procainamide. Clin. Pharmacol. Ther. 20:278-289.

11. Benowitz, N. L. 1986. Clinical pharmacology of nicotine. Annu. Rev. Med. 37:21-32.

12. Su, C. 1982. Actions of nicotine and smoking on circulation. Pharmacol. \& Ther. 17:129-141.

13. Comroe, J. H. 1960. The pharmacologic actions of nicotine. Ann. N. Y. Acad. Sci. 90:48-51.

14. Stalhandske, T. 1970. Effects of increased liver metabolism of nicotine on its uptake, elimination and toxicity in mice. Acta Physiol. Scand. 80:222-234.

15. Oldendorf, W. H. 1974. Lipid solubility and drug penetration of the blood brain barrier. Proc. Soc. Exp. Biol. Med. 147:813-816.

16. Greenblatt, D. J., B. L. Ehrenberg, J. S. Harmatz, J. Gunderman, J. M. Scavone, and R. I. Shader. 1987. Determinants of benzodiazepine dynamics after single i.v. doses. Clin. Pharmacol. Ther. 41:243 (Abstr.).

17. Wagner, J. C. 1974. A safe method for rapidly achieving plasma concentration plateaus. Clin. Pharmacol. Ther. 16:691-700. 
18. Jacob, P., III, M. Wilson, and N. L. Benowitz. 1981. Improved gas chromatographic method for the determination of nicotine and cotinine in biologic fluids. J. Chromatogr. Biomed. Appl. 222:61-70.

19. Tur, E., R. H. Guy, M. Tur, and H. I. Maibach. 1983. Noninvasive assessment of local nicotinate pharmacodynamics by photoplethysmography. J. Invest. Dermatol. 80:499-503.

20. Katz, M., and M. M. Sokal. 1980. Skin perfusion in pregnancy. Am. J. Obstet. Gynecol. 137:30-33.

21. Peck, C. C., S. L. Beal, L. B. Sheiner, and A. I. Nichols. 1984. Extended least squares nonlinear regression: a possible solution to the "choice of weights" problem in analysis of individual pharmacokinetic data. J. Pharmacokinet. Biopharm. 12:545-558.

22. Neutze, J. M., F. Wyler, and A. W. Rudolph. 1968. Use of radioactive microspheres to assess distribution of cardiac output in rabbits. Am. J. Physiol. 215:486-495.

23. Frank, D. W. 1976. Physiological data of laboratory animals. In Handbook of Laboratory Animal Science. E. C. Melby and N. H. Altman, editors. CRC Press, Inc., Boca Raton, Florida. vol. III. 23-64.

24. Gibaldi, M., and D. Perrier. 1982. Pharmacokinetics. Marcel Dekker, Inc., New York. 494 pp.

25. Ganong, W. F. 1975. Review of Medical Physiology. Lange, Medical Publications, Los Altos, California. 7th ed. 446 pp.

26. Schmiterlow, C. G., E. Hansson, G. Anderson, L. E. Applegren, and P. C. Hoffman. 1967. Distribution of nicotine in central nervous system. Ann. N. Y. Acad. Sci. 142:2-14.
27. Galeazzi, R. L. 1985. Pharmacodynamics, pharmacokinetics, or both? In Pharmacokinetics and Pharmacodynamics of Psychoactive Drugs. G. Barnett and C. N. Chiang, editors. Biomedical Publications, Foster City, California. 169-184.

28. Jamrozik, K., G. Fowler, M. Vessey, and N. Wald. 1984. Placebo controlled trial of nicotine chewing gum in general practice. $\mathrm{Br}$. Med. J. 289:1782-1785.

29. Chow, M. J., J. J. Ambre, T. I. Ruo, A. J. Atkinson, D. J. Bowsher, and M. W. Fischman. 1985. Kinetics of cocaine distribution, elimination, and chronotropic effects. Clin. Pharmacol. Ther. 38:318324.

30. Harger, R. N., and R. B. Forney. 1963. The aliphatic alcohols. A. Stolman, editor. In Progress in Chemical Toxicology. Academic Press, New York. Vol. 1. 53-134.

31. Kalant, H., A. E. Leblanc, and R. J. Gibbins. 1971. Tolerance to, and dependence on, some nonopiate psychotropic drugs. Pharmacol. Rev. 23:135-191.

32. Russell, M. A. H., and C. Feyerabend. 1978. Cigarette smoking: a dependence on high-nicotine boli. Drug Metab. Rev. 8:29-57.

33. Marks, M. J., E. Romm, D. K. Gaffney, and A. C. Collins. 1986. Nicotine-induced tolerance and receptor changes in four mouse strains. J. Pharmacol. Exp. Ther. 237:809-819.

34. Lee, B. L., N. L. Benowitz, and P. Jacob, III. 1987. Influence of tobacco abstinence on the disposition kinetics and effects of nicotine. Clin. Pharmacol. Ther. 41:474-479. 